\title{
Efecto del tipo de solvente y tamaño de partícula en la determinación de pH en compost
}

\section{Effect of the type of solvent and particle size in the determination of $\mathrm{pH}$ in compost}

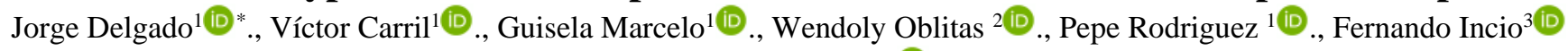
y Rolando Romero 4 (D)

\section{RESUMEN}

El objetivo de esta investigación fue evaluar la metodología para la medición del potencial de iones hidrógeno $(\mathrm{pH})$ en el compost. El diseño fue experimental, ya que a través de experimentos se pretendió llegar a la causa de un fenómeno, en ese sentido el $\mathrm{pH}$, fue sometido a la influencia del volumen y tipo de extractante y el tamaño de partícula. Se encontró las relaciones de dependencia del valor del pH a partir de la variación del tamaño de partícula, el incremento de volumen del solvente, concentración y tipo de solvente. Los tamaños de partícula ensayados fueron de 0.25, 0.5, 1.0 y $2.0 \mathrm{~mm}$. Los solventes empleados fueron agua, cloruro de potasio $(\mathrm{KCl})$ y Cloruro de calcio dihidratado $\left(\mathrm{CaCl}_{2} .2 \mathrm{H}_{2} \mathrm{O}\right)$ al 0.01 y 0.1molar. Se realizaron 100 mediciones de $\mathrm{pH}$. Los resultados mostraron independencia cuando las condiciones de trabajo fueron 10 gramos de compost, tamaños de partícula entre 1.0 y $2.0 \mathrm{~mm}$, en volúmenes de 100, 125 y $150 \mathrm{ml}$ de $\mathrm{CaCl}_{2} .2 \mathrm{H}_{2} \mathrm{O}$ de concentración 0.01 molar. Se concluye que cuando se emplea volúmenes entre 100 y $125 \mathrm{ml}$ del $\mathrm{CaCl}_{2}$ 0.01M, con tamaños de partícula de compost entre 1 y $2 \mathrm{~mm}$, los valores del pH, tiende a ser homogéneos.

Palabras clave: Solvente, partícula, cloruro de calcio dihidratado.

\begin{abstract}
The objective of this research was to evaluate the methodology for measuring the hydrogen ion potential $(\mathrm{pH})$ in compost. The design was experimental, since through experiments it was intended to reach the cause of a phenomenon, in that sense the $\mathrm{pH}$ was subjected to the influence of the volume and type of extractant and the particle size. The dependence of the $\mathrm{pH}$ value on the variation of the particle size, the increase in solvent volume, concentration and type of solvent was found. The particle sizes tested were $0.25,0.5,1.0$ and $2.0 \mathrm{~mm}$. The solvents used were water, potassium chloride $(\mathrm{KCl})$ and calcium chloride dihydrate $\left(\mathrm{CaCl}_{2} .2 \mathrm{H}_{2} \mathrm{O}\right)$ at 0.01 and 0.1 molar. A total of $100 \mathrm{pH}$ measurements were performed. The results showed independence when the working conditions were 10 grams of compost, particle sizes between 1.0 and $2.0 \mathrm{~mm}$, in volumes of 100,125 and $150 \mathrm{ml}$ of $\mathrm{CaCl}_{2} .2 \mathrm{H}_{2} \mathrm{O}$ of 0.01 molar concentration. It is concluded that when volumes between 100 and $125 \mathrm{ml}$ of $0.01 \mathrm{M} \mathrm{CaCl}_{2} .2 \mathrm{H}_{2} \mathrm{O}$ are used, with compost particle sizes between 1 and $2 \mathrm{~mm}$, the $\mathrm{pH}$ values tend to be homogeneous.
\end{abstract}

Keywords: Solvent, particle, calcium chloride dihydrate.

DOI: https://doi.org/10.37787/pakamuros-unj.v9i3.214

Recibido: 16/02/2021. Aceptado:02/06/2021

* Autor para correspondencia

\footnotetext{
1. Universidad Nacional de Jaén, Perú. Email: jorge_delgado@unj.edu.pe; viceinvestigacion@unj.edu.pe; guiseeg@gmail.com; bmzluis@gmail.com.

2. Universidad Nacional de Cajamarca, Perú. Email: woblitasp_epg19@unc.edu.pe

3. Universidad Nacional Intercultural Fabiola Salazar Leguía de Bagua, Perú. Email: fincio@unibagua.edu.pe

4. Universidad Tecnológica del Perú. Email: e15138@utp.edu.pe
} 


\section{INTRODUCCIÓN}

El pH representa uno de los principales parámetros fisicoquímicos que es determinado en materiales compostados (Iqbal et al., 2010). En el caso del compost, no se podría emplear la relación que se emplea en el suelo, debido a su elevada concentración de materia orgánica que tiende a formar una masa heterogénea, donde el bulbo del electrodo no operará con funcionalidad, al emplear agua, el valor del pH se eleva, debido a los iones hidrógeno presentes que se diluyen, modificando el valor del pH (Tan, 1993, pp. 267-271)

Millán, et al (2017) manifiestan que, no existe un acuerdo general para emplear determinado volumen o tipo de extractante, existen autores que para medir del $\mathrm{pH}$ emplean relaciones de 10 gramos de compost en $50 \mathrm{ml}$ de solvente, sin mencionar alguna justificación. Sepúlveda y Salinas (2014) indican que, para la medición del pH del sustrato humificado emplearon la relación suelo: agua 1:2.5, tamaño de partícula 2 mm (p. 96). Florida y Pocomucha (2016) determinaron el pH empleando una relación de 10 gramos de compost en 25 mililitros de agua, empleando un pHmetro. Bohorquez, (2014) manifiestan haber realizado mediciones de $\mathrm{pH}$ en compost obtenido de residuos de la producción de caña de azúcar, utilizando 10 gramos de compost en 10 mililitros de agua. Rivas y Silva (2020) señalan haber cuantificado el pH de compost elaborado de diversos residuos como tegumentos de café, hojas de árboles, empleando la relación 1:2 basándose en la norma técnica colombiana (NTC2014) (p.89).

Van Erp et al., (2001) emplearon cloruro de calcio 0.01M, para extraer multinutrientes, además del pH. Las sales, como el cloruro de potasio o el cloruro de calcio no provoca exceso de iones hidrógeno o de iones oxhidrilo, debido a que estas sales son completamente neutras. Barrena (2006) indica que, el pH se determina sobre el extracto acuso de una muestra fresca de compost, empleando las proporciones 1/5. Con base en lo mencionado, se evaluó la medición del pH del compost, a partir de variaciones establecidas en el tamaño de partícula, la relación compost solvente, el tipo y concentración de solvente.

\section{MATERIALES Y MÉTODOS}

\section{Tipo y diseño de la investigación}

Tipo explicativo, ya que se trató de explicar el efecto del tipo de solvente y tamaño de partícula en la determinación de $\mathrm{pH}$ en compost. Diseño experimental, ya que a través de experimentos se pretendió llegar a la causa de un fenómeno. $\mathrm{El} \mathrm{pH}$, fue sometido a la influencia del volumen y tipo de extractante y el tamaño de partícula. 


\section{Método}

Se trabajaron con los solventes y concentraciones: agua, cloruro de potasio (KCl 0.01 y 0.1 molar) y cloruro de calcio dihidratado $\left(\mathrm{CaCl}_{2} .2 \mathrm{H}_{2} \mathrm{O} 0.01\right.$ y 0.1 molar). Las mediciones fueron realizadas en las relaciones compost solvente 10/50, 10/75, 10/100, 10/125 y 10/150 gramos de compost por mililitro de solvente. Los tamaños de partícula fueron 0.25, 0.50, 1.0, $2.0 \mathrm{~mm}$. Se llevaron a cabo 100 mediciones de pH, mediante el empleo de soluciones de marca HANNA. El medidor de pH fue demodelo HI 991003 con electrodo de titanio y el multiparámetro modelo HI 2550 ambos calibrados en tres puntos con las soluciones buffers, de la marca HANNA.

El procesamiento de datos se realizó a través de un diseño factorial entre dos factores, con la finalidad de encontrar el grupo de resultados que pueda tener un promedio diferente, luego se procedió a considerar las comparaciones: entre los solventes y el volumen, entre los solventes y el pH y entre el volumen y el $\mathrm{pH}$, evaluándose los grupos que generaban las diferencias, también se utilizó el coeficiente de variación, para hallar resultados homogéneos, todos los análisis estadísticos fueron procesados mediante el SPSS.

\section{RESULTADOS}

Tabla 1, se indica los resultados basados en pruebas de hipótesis de comparación de promedios por bloques, para el $\mathrm{pH}$, donde el factor 1 fue el solvente y el factor 2 fue el volumen. Existiendo diferencia entre los promedios de los resultados, de acuerdo con tipos de solvente, incremento de volumen y en la interacción de tipo de solvente e incremento de volumen.

Tabla 1. Análisis de varianza para el incremento de volumen y el tipo de solvente

\begin{tabular}{lccccc}
\hline Origen & $\begin{array}{c}\text { Tipo III de } \\
\text { suma de } \\
\text { cuadrados }\end{array}$ & gl & $\begin{array}{c}\text { Media } \\
\text { cuadrática }\end{array}$ & F & Sig. \\
\hline Modelo & $8873.511^{\mathrm{a}}$ & 25 & 354.94 & 56143.693 & 0 \\
volumen & 0.394 & 4 & 0.098 & 15.561 & 0 \\
solvente & 37.567 & 4 & 9.392 & 1485.558 & 0 \\
volumen * & 0.678 & 16 & 0.042 & 6.706 & 0 \\
solvente & 0.474 & 75 & 0.006 & & \\
Error & 8873.985 & 100 & & & \\
Total & & & & \\
\hline
\end{tabular}

La Tabla 2, indica las pruebas de comparaciones múltiples de medias entre solventes, resultando por lo menos una diferencia entre las medias de los tratamientos de los grupos (solventes), donde los resultados 
obtenidos para el promedio de agua y el $\mathrm{KCl} 0.01 \mathrm{M}$ son iguales $(\mathrm{p}>0.05)$ y el resto de los promedios entre par de solventes son diferentes.

Tabla 2. Comparación múltiple de medias entre grupos de solvente

\begin{tabular}{|c|c|c|c|c|c|c|}
\hline \multirow{2}{*}{ (I) solvente } & & \multirow{2}{*}{$\begin{array}{l}\text { Diferencia de } \\
\text { medias (I-J) }\end{array}$} & \multirow{2}{*}{ Desv. Error } & \multirow{2}{*}{ Sig. } & \multicolumn{2}{|c|}{ Intervalo de confianza al $95 \%$} \\
\hline & & & & & Límite inferior & Límite superior \\
\hline \multirow{4}{*}{ Agua } & $\mathrm{KCl} 0.01 \mathrm{M}$ & -0.002 & 0.02514 & 1 & -0.0814 & 0.0774 \\
\hline & $\mathrm{KCl} 0.1 \mathrm{M}$ & $0.2165^{*}$ & 0.02514 & 0 & 0.1371 & 0.2959 \\
\hline & $\mathrm{CaCl}_{2} 0.01 \mathrm{M}$ & $0.4040^{*}$ & 0.02514 & 0 & 0.3246 & 0.4834 \\
\hline & $\mathrm{CaCl}_{2} 0.1 \mathrm{M}$ & $1.6395^{*}$ & 0.02514 & 0 & 1.5601 & 1.7189 \\
\hline \multirow{4}{*}{ KCl0.01M } & Agua & 0.002 & 0.02514 & 1 & -0.0774 & 0.0814 \\
\hline & $\mathrm{KCl} 0.1 \mathrm{M}$ & $0.2185^{*}$ & 0.02514 & 0 & 0.1391 & 0.2979 \\
\hline & $\mathrm{CaCl}_{2} 0.01 \mathrm{M}$ & $0.4060^{*}$ & 0.02514 & 0 & 0.3266 & 0.4854 \\
\hline & $\mathrm{CaCl}_{2} 0.1 \mathrm{M}$ & $1,6415^{*}$ & 0.02514 & 0 & 1.5621 & 1.7209 \\
\hline \multirow{4}{*}{$\mathrm{KCl} 0.1 \mathrm{M}$} & Agua & $-2165^{*}$ & 0.02514 & 0 & -0.2959 & -0.1371 \\
\hline & $\mathrm{KCl} 0.01 \mathrm{M}$ & $-2185^{*}$ & 0.02514 & 0 & -0.2979 & -0.1391 \\
\hline & $\mathrm{CaCl}_{2}$ 0.01M & $0.1875^{*}$ & 0.02514 & 0 & 0.1081 & 0.2669 \\
\hline & $\mathrm{CaCl}_{2} 0.1 \mathrm{M}$ & $1.4230^{*}$ & 0.02514 & 0 & 1.3436 & 1.5024 \\
\hline \multirow{4}{*}{$\mathrm{CaCl}_{2} 0.01 \mathrm{M}$} & Agua & $-4040^{*}$ & 0.02514 & 0 & -0.4834 & -0.3246 \\
\hline & $\mathrm{KCl} 0.01 \mathrm{M}$ & $-4060^{*}$ & 0.02514 & 0 & -0.4854 & -0.3266 \\
\hline & $\mathrm{KCl} 0.1 \mathrm{M}$ & $-1875^{*}$ & 0.02514 & 0 & -0.2669 & -0.1081 \\
\hline & $\mathrm{CaCl}_{2} 0.1 \mathrm{M}$ & $1.2355^{*}$ & 0.02514 & 0 & 1.1561 & 1.3149 \\
\hline \multirow{4}{*}{$\mathrm{CaCl}_{2} 0.1 \mathrm{M}$} & Agua & $-1.6395^{*}$ & 0.02514 & 0 & -1.7189 & -1.5601 \\
\hline & KCl0.01M & $-1.6415^{*}$ & 0.02514 & 0 & -1.7209 & -1.5621 \\
\hline & $\mathrm{KCl} 0.1 \mathrm{M}$ & $-1.4230^{*}$ & 0.02514 & 0 & -1.5024 & -1.3436 \\
\hline & $\mathrm{CaCl}_{2} 0.01 \mathrm{M}$ & $-1.2355^{*}$ & 0.02514 & 0 & -1.3149 & -1.1561 \\
\hline
\end{tabular}

*existe diferencias significativas

La Tabla 3, muestra los agrupamientos por promedios homogéneos para los solventes agua y KCl 0.01M. 
Tabla 3. Agrupamiento de Solventes por promedios iguales

\begin{tabular}{lccccc}
\hline \multirow{2}{*}{ Solvente } & $\mathbf{N}$ & \multicolumn{5}{c}{ Subconjunto } \\
\cline { 3 - 6 } & & $\mathbf{1}$ & $\mathbf{2}$ & $\mathbf{3}$ & $\mathbf{4}$ \\
\hline $\mathrm{CaCl}_{2} 0.1 \mathrm{M}$ & 20 & 8.2115 & & & \\
$\mathrm{CaCl}_{2} 0.01 \mathrm{M}$ & 20 & & 9.447 & & \\
$\mathrm{KCl} 0.1 \mathrm{M}$ & 20 & & & 9.6345 & \\
Agua & 20 & & & & 9.851 \\
KCl0.01M & 20 & & & & 9.853 \\
Sig. & & 1 & 1 & 1 & 1 \\
\hline
\end{tabular}

En la Tabla 4, indica que existen diferencias significativas a un 0.05 entre los promedios de resultados, de acuerdo con el solvente y el pH son iguales entre los en la interacción. Al comparar los resultados entre promedios en los grupos de volumen, resultan ser iguales en todos los casos, salvo para los volúmenes que se encuentra entre 75 y 125 los promedios fueron diferentes.

Tabla 4. Comparación múltiple de medias entre volúmenes de solventes

\begin{tabular}{ccccccc}
\hline (I) volumen & Diferencia de & Desv. Error & Sig. & \multicolumn{2}{c}{ Intervalo de confianza al 95\% } \\
\cline { 5 - 6 } & & & & $\begin{array}{c}\text { Límite } \\
\text { inferior }\end{array}$ & $\begin{array}{c}\text { Límite } \\
\text { superior }\end{array}$ \\
\hline 50 & 75 & -0.0775 & 0.02514 & 0.060 & -0.1569 & 0.0019 \\
& 100 & $-1330^{*}$ & 0.02514 & 0.000 & -0.2124 & -0.0536 \\
& 125 & $-1585^{*}$ & 0.02514 & 0.000 & -0.2379 & -0.0791 \\
& 150 & $-1705^{*}$ & 0.02514 & 0.000 & -0.2499 & -0.0911 \\
\hline 75 & 50 & 0.0775 & 0.02514 & 0.060 & -0.0019 & 0.1569 \\
& 100 & -0.0555 & 0.02514 & 0.310 & -0.1349 & 0.0239 \\
& 125 & $-0810^{*}$ & 0.02514 & 0.043 & -0.1604 & -0.0016 \\
& 150 & $-0930^{*}$ & 0.02514 & 0.013 & -0.1724 & -0.0136 \\
\hline 100 & 50 & $0.1330^{*}$ & 0.02514 & 0.000 & 0.0536 & 0.2124 \\
& 75 & 0.0555 & 0.02514 & 0.310 & -0.0239 & 0.1349 \\
& 125 & -0.0255 & 0.02514 & 0.904 & -0.1049 & 0.0539 \\
& 150 & -0.0375 & 0.02514 & 0.695 & -0.1169 & 0.0419 \\
\hline 125 & 50 & $0.1585^{*}$ & 0.02514 & 0.000 & 0.0791 & 0.2379 \\
& 75 & $0.0810^{*}$ & 0.02514 & 0.043 & 0.0016 & 0.1604 \\
& 100 & 0.0255 & 0.02514 & 0.904 & -0.0539 & 0.1049 \\
& 150 & -0.0120 & 0.02514 & 0.994 & -0.0914 & 0.0674 \\
\hline 150 & 50 & $0.1705^{*}$ & 0.02514 & 0.000 & 0.0911 & 0.2499 \\
& 75 & $0.0930^{*}$ & 0.02514 & 0.013 & 0.0136 & 0.1724 \\
& 100 & 0.0375 & 0.02514 & 0.695 & -0.0419 & 0.1169 \\
& 125 & 0.0120 & 0.02514 & 0.994 & -0.0674 & 0.0914 \\
\hline
\end{tabular}

La Tabla 5, muestra tres grupos de promedio de resultados homogéneos, para el incremento de volumen. El primer grupo de 50 y $75 \mathrm{ml}$, segundo grupo de 75 y $100 \mathrm{ml}$ y el tercer grupo 100, 125 y $150 \mathrm{ml}$. 
Tabla 5. Agrupamiento de volumen por promedios iguales

\begin{tabular}{lllccc}
\hline \multirow{2}{*}{ Volumen } & \multirow{2}{*}{$\mathbf{N}$} & \multicolumn{3}{c}{ Subconjunto } \\
\cline { 3 - 6 } & & & $\mathbf{1}$ & $\mathbf{2}$ & $\mathbf{3}$ \\
\hline & 50 & 20 & 9.2915 & & \\
& 75 & 20 & 9.369 & 9.369 & \\
& 100 & 20 & & 9.4245 & 9.4245 \\
& 125 & 20 & & & 9.45 \\
& 150 & 20 & & & 9.462 \\
Sig. & & & 0.06 & 0.31 & 0.695 \\
\hline
\end{tabular}

La Figura 1 indica que los grupos difieren entre sí. En el caso del cloruro de calcio a menor concentración $\left(\mathrm{CaCl}_{2}\right.$ 0.01M) empleando volúmenes de 75, 100 y $125 \mathrm{ml}$, demuestra homogeneidad de valores, ya que no presenta dependencia respecto al aumento del volumen de solvente, en ese intervalo. Sin embargo, el cloruro de calcio a mayor concentración $\left(\mathrm{CaCl}_{2} 0.1 \mathrm{M}\right)$, presenta valores de $\mathrm{pH}$ inferiores al resto, con tendencia a la disminución.

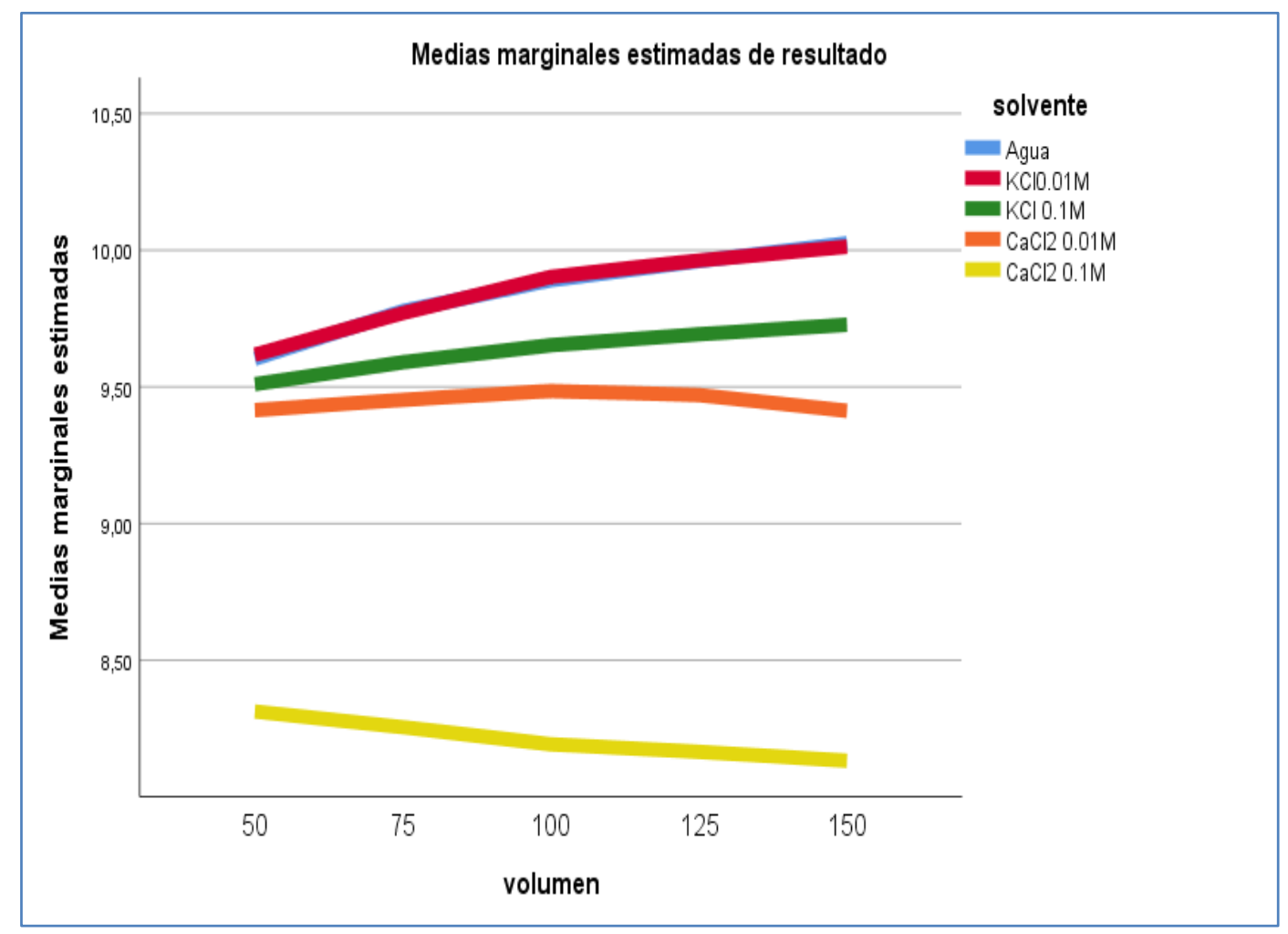

Figura 1. Representación de promedios de resultados entre los solventes y su volumen 
La Tabla 6, muestra la varianza de dos factores entre tipo de solvente y $\mathrm{pH}$, observamos que al menos uno de los promedios es diferente al resto. Sin embargo, en la interacción de ambas no hay diferencia significativa entre sus promedios.

Tabla 6. Análisis de varianza para el tipo de solvente y pH

\begin{tabular}{lccccc}
\hline \multicolumn{1}{c}{ Origen } & $\begin{array}{c}\text { Tipo III de } \\
\text { suma de } \\
\text { cuadrados }\end{array}$ & Gl & $\begin{array}{c}\text { Media } \\
\text { cuadrática }\end{array}$ & F & Sig. \\
\hline Modelo & $8872.795^{\mathrm{a}}$ & 20 & 443.64 & 29839.568 & 0 \\
$\mathrm{pH}$ & 0.2 & 3 & 0.067 & 4.491 & 0.006 \\
solvente & 37.567 & 4 & 9.392 & 631.693 & 0 \\
pH ${ }^{*}$ & 0.156 & 12 & 0.013 & 0.876 & 0.574 \\
solvente & 1.189 & 80 & 0.015 & & \\
Error & 8873.985 & 100 & & & \\
Total & & & & & \\
\hline
\end{tabular}

La Tabla 7, indica la comparación de media entre tipos de $\mathrm{pH}$ a diferentes tamaños de partícula, se observa que, al comparar por par de tipos de $\mathrm{pH}$, entre el $\mathrm{pH}$ medido a un tamaño de partícula de 0.25 mm difiere con el pH medido a $0.5 \mathrm{~mm}$ y $1 \mathrm{~mm}$, en los demás casos no hay diferencia significativa entre sus promedios.

Tabla 7. Comparación múltiple de medias entre pH y tamaños de partículas

\begin{tabular}{|c|c|c|c|c|c|c|}
\hline \multirow{2}{*}{ (I) $\mathrm{pH}$} & & \multirow{2}{*}{$\begin{array}{l}\text { Diferencia de } \\
\text { medias (I-J) }\end{array}$} & \multirow{2}{*}{$\begin{array}{l}\text { Desv. } \\
\text { Error }\end{array}$} & \multirow{2}{*}{ Sig. } & \multicolumn{2}{|c|}{$\begin{array}{c}\text { Intervalo de confianza al } \\
95 \%\end{array}$} \\
\hline & & & & & $\begin{array}{l}\text { Límite } \\
\text { inferior }\end{array}$ & $\begin{array}{l}\text { Límite } \\
\text { superior }\end{array}$ \\
\hline \multirow{3}{*}{ pH 0.25} & $\mathrm{pH} 0.5$ & $-1172^{*}$ & 0.0345 & 0.013 & -0.2157 & -0.0187 \\
\hline & pH 1 & $-0992^{*}$ & 0.0345 & 0.048 & -0.1977 & -0.0007 \\
\hline & pH 2 & -0.0644 & 0.0345 & 0.329 & -0.1629 & 0.0341 \\
\hline \multirow{3}{*}{ pH 0.5} & pH 0.25 & $0,1172^{*}$ & 0.0345 & 0.013 & 0.0187 & 0.2157 \\
\hline & pH 1 & 0.018 & 0.0345 & 0.965 & -0.0805 & 0.1165 \\
\hline & pH 2 & 0.0528 & 0.0345 & 0.508 & -0.0457 & 0.1513 \\
\hline \multirow{3}{*}{ pH 1} & pH 0.25 & $0.0992^{*}$ & 0.0345 & 0.048 & 0.0007 & 0.1977 \\
\hline & pH 0.5 & -0.018 & 0.0345 & 0.965 & -0.1165 & 0.0805 \\
\hline & $\mathrm{pH} 2$ & 0.0348 & 0.0345 & 0.797 & -0.0637 & 0.1333 \\
\hline \multirow{3}{*}{ pH 2} & $\mathrm{pH} 0.25$ & 0.0644 & 0.0345 & 0.329 & -0.0341 & 0.1629 \\
\hline & pH 0.5 & -0.0528 & 0.0345 & 0.508 & -0.1513 & 0.0457 \\
\hline & pH 1 & -0.0348 & 0.0345 & 0.797 & -0.1333 & 0.0637 \\
\hline
\end{tabular}

En la Tabla 8, se muestra igualdad entre dos grupos de promedio respecto al tamaño de partícula, 0.25 , $2 \mathrm{~mm}, 2$, 1 y $0.5 \mathrm{~mm}$ respectivamente. 
Tabla 8. Agrupamiento de $\mathrm{pH}$ por promedios iguales

\begin{tabular}{llcc}
\hline \multirow{2}{*}{$\mathbf{p H}$} & \multirow{2}{*}{} & \multicolumn{2}{c}{ Subconjunto } \\
\cline { 3 - 4 } & & $\mathbf{1}$ & $\mathbf{2}$ \\
\hline pH 0.25 & 25 & 9.3292 & \\
pH 2 & 25 & 9.3936 & 9.3936 \\
pH 1 & 25 & & 9.4284 \\
pH 0.5 & 25 & & 9.4464 \\
Sig. & & 0.329 & 0.508 \\
\hline
\end{tabular}

La Figura 2, muestra que el tamaño de partícula, al emplear agua y cloruro de potasio a baja concentración (KCl $0.01 \mathrm{M})$ produce valores de $\mathrm{pH}$ similares, mayores a los obtenidos con el cloruro de potasio a elevada concentración ( $\mathrm{KCl} 0.1 \mathrm{M}$ ) y el $\mathrm{CaCl}_{2} .2 \mathrm{H}_{2} \mathrm{O}$ en ambas concentraciones. En el caso de emplear cloruro de potasio de baja concentración $\left(\mathrm{CaCl}_{2} .2 \mathrm{H}_{2} \mathrm{O} 0.01 \mathrm{M}\right)$, demuestra igualdad de promedio entre los resultados, a tamaño de partícula 0.5, 1 y 2 mm respectivamente. Cuando se emplea cloruro de calcio de mayor concentración $\left(\mathrm{CaCl}_{2} \cdot 2 \mathrm{H}_{2} \mathrm{O} 0.1 \mathrm{M}\right)$ el tamaño de partícula demuestra independencia.

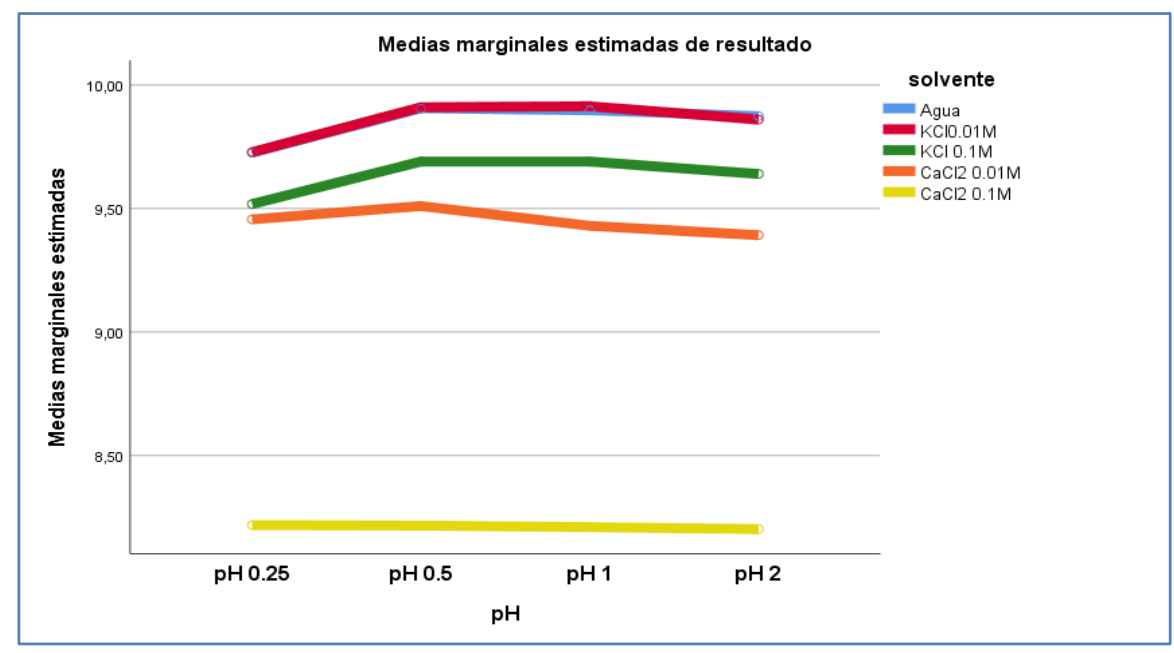

Figura 2. Representación de promedios de resultados entre los solventes y su pH

La Tabla 9 de análisis de varianza de dos factores entre incremento de volumen y $\mathrm{pH}$, se observa que existe diferencia entre los promedios de resultados. Los resultados de la tabla están fundamentados en la prueba de efectos pH-volumen. Los resultados han sido estimados para un coeficiente de terminación $\left(\mathrm{R}^{2}=0.996\right)$ las medias, con un error de media cuadrática de 0.006, con un ajuste de 0.995. 
Tabla 9. Análisis de varianza para pH e incremento de volumen

\begin{tabular}{cccccc}
\hline Origen & $\begin{array}{c}\text { Tipo III de } \\
\text { suma de } \\
\text { cuadrados }\end{array}$ & gl & $\begin{array}{c}\text { Media } \\
\text { cuadrática }\end{array}$ & F & Sig. \\
\hline Modelo & $8835.528^{\mathrm{a}}$ & 20 & 441.776 & 919.017 & 0 \\
$\mathrm{pH}$ & 0.2 & 3 & 0.067 & 0.139 & 0.936 \\
volumen & 0.394 & 4 & 0.098 & 0.205 & 0.935 \\
$\mathrm{pH}^{*}$ & 0.063 & 12 & 0.005 & 0.011 & 1 \\
volumen & 38.456 & 80 & 0.481 & & \\
Error & 8873.985 & 100 & & & \\
Total & & & & & \\
\hline
\end{tabular}

La Figura 3, indica que los grupos iguales están definidos en la interacción del incremento de volumen de los solventes y el tamaño de partícula, obteniendo valores similares de $\mathrm{pH}$, entre tamaños de partícula de 1 y 2.00 mm, para el volumen de 100, 125 y 150 ml los pH son homogéneos.

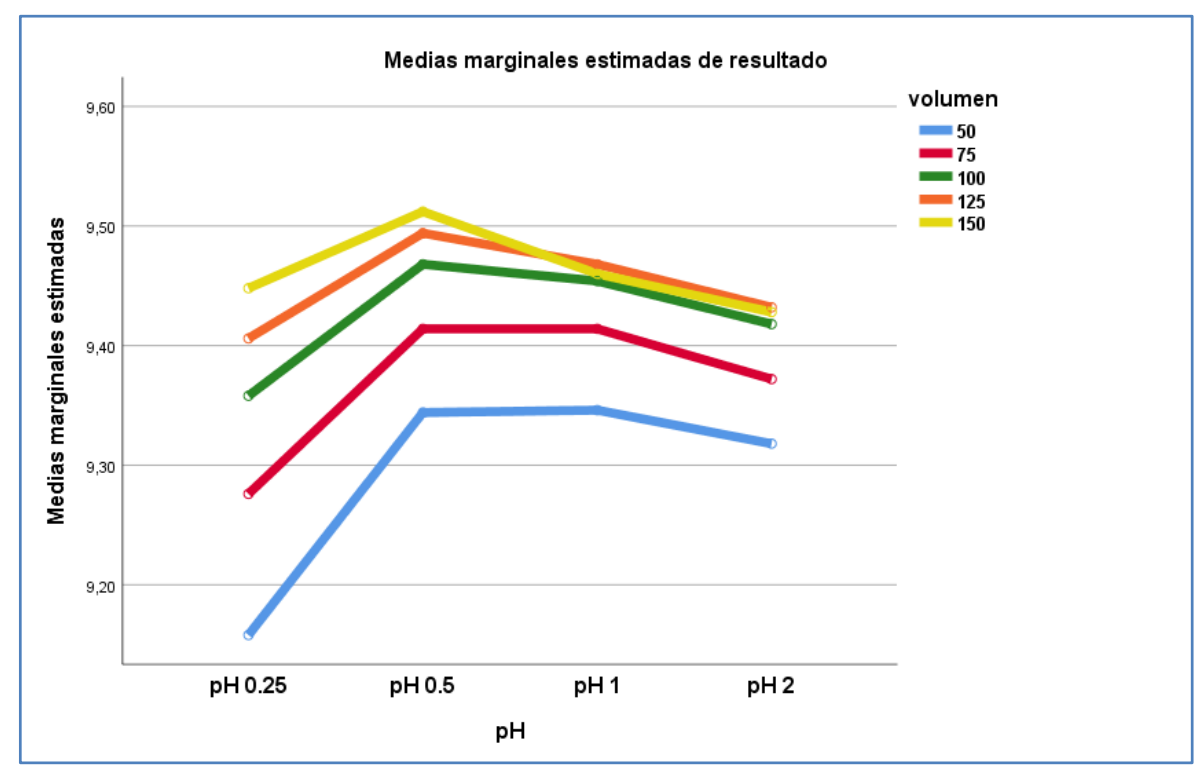

Figura 3. Representación de promedios de resultados entre el volumen y su pH

La Tabla 10, se observa que cuando se evaluó el $\mathrm{pH}$ a un tamaño de partícula de $0.25 \mathrm{~mm}$ dio valores homogéneos. Mientras que los tamaños de partícula entre 0.5, 1 y $2.0 \mathrm{~mm}$, condujo a la obtención de valores homogéneos 6.82, 6.92 y 6.90\% respectivamente. 
Tabla 10. Coeficiente de variación del pH frente al tamaño de partícula

\begin{tabular}{cccccc}
\hline Variable & Tamaño & N & Media & Desviación estándar & Coeficiente de variación \\
\hline $\mathrm{pH}$ & 0.25 & 25 & 9.331 & 0.597 & 6.40 \\
& 0.50 & 25 & 9.447 & 0.654 & 6.92 \\
& 1.00 & 25 & 9.427 & 0.651 & 6.90 \\
& 2.00 & 25 & 9.393 & 0.641 & 6.82 \\
\hline
\end{tabular}

La Tabla 11, establece la variabilidad del $\mathrm{pH}$ a medida que se aumenta el volumen del solvente, se mantiene constante el peso del compost, encontrado similitud entre los coeficientes de variación de 100 y $125 \mathrm{ml}$.

Tabla 11. Coeficiente de variación para el caso del pH respecto al volumen de solvente

\begin{tabular}{cccccc}
\hline Variable & Volumen & N & Media & Desviación estándar & Coeficiente de variación \\
\hline $\mathrm{pH}$ & 50 & 20 & 9.291 & 0.518 & 5.57 \\
& 75 & 20 & 9.368 & 0.590 & 6.30 \\
& 100 & 20 & 9.424 & 0.655 & 6.95 \\
& 125 & 20 & 9.448 & 0.688 & 7.28 \\
& 150 & 20 & 9.462 & 0.722 & 7.63 \\
\hline
\end{tabular}

La Tabla 12, se aprecia los resultados de la evaluación del coeficiente de variación del pH respecto al tipo de solvente, el menor coeficiente de variación lo produce el $\mathrm{CaCl}_{2} 2 \mathrm{H}_{2} \mathrm{O}$ a menor concentración

Tabla 12. Coeficiente de variación para el caso del pH respecto al tipo de solvente

\begin{tabular}{cccccc}
\hline Variable & Solvente & $\mathbf{N}$ & Media & Desviación estándar & Coeficiente Variación \\
\hline $\mathrm{pH}$ & Agua & 20 & 9.8503 & 0.1740 & 1.77 \\
& $\mathrm{CaCl}_{2} 0.01 \mathrm{M}$ & 20 & 9.4470 & 0.0680 & 0.72 \\
& $\mathrm{CaCl}_{2} 0.1 \mathrm{M}$ & 20 & 8.2105 & 0.0685 & 0.83 \\
& $\mathrm{KCl} 0.01 \mathrm{M}$ & 20 & 9.8527 & 0.1680 & 1.71 \\
& $\mathrm{KCl} 0.1 \mathrm{M}$ & 20 & 9.6335 & 0.1154 & 1.20 \\
\hline
\end{tabular}

\section{DISCUSIÓN}

Los valores de $\mathrm{pH}$ obtenidos con el $\mathrm{CaCl}_{2}$ 0.01M a menor concentración, empleando volúmenes de 75 , 100 y 125 ml, demuestran homogeneidad. Resultados similares fueron encontrados por Millán et al (2017), quienes determinaron homogeneidad en las muestras, acercándose a la realidad de las muestras. 
Empleando cloruro de calcio 0.01 molar la medición del pH no depende del incremento de volumen entre 75 y 125 mililitros. Sin embargo, cuando se emplean el agua, $\mathrm{KCl}$ y $\mathrm{CaCl}_{2}$ a 0.1 molar el valor de la medición se incrementa. En consecuencia, la medición debe realizarse donde el pH produzca valores homogéneos, ya que la medición será independiente de la cantidad de extractante. Al respecto los mismos autores manifiestan que, esto ocurre en relaciones compost/extractante mayor a la relación 10 gramos de compost en 50 mililitros de $\mathrm{CaCl}_{2} 0.01$ molar. Igualmente, el pH medido en el agua es menor que el pH medido $\mathrm{CaCl}_{2}$, esto significa que esta sal extrae mejor los iones hidrógeno en una solución, acercándose a la realidad de la muestra.

Queda demostrado que los coeficientes de variación aumentan progresivamente a medida que el volumen del solvente se incrementa. Al respecto Delgado et al, (2019) manifiestan que, para suelos ácidos, los cuales son de naturaleza arcillosa, el incremento de volumen del solvente influye directamente en la medición del pH, incrementándolo. La materia orgánica, en solución tiene comportamiento de coloidal, tal como las arcillas (Lerma et al., 2015). Es por ello que el comportamiento del pH es similar, entre suspensiones de suelos arcillosos y suspensiones de compost.

En la Tabla 9 se observó que el resultado de la medición del pH medido con $\mathrm{CaCl}_{2}$ 0.01M, se obtuvo el menor coeficiente de variación 0.72. Por lo que se puede inferir que este solvente podría ser considerados para la medición del pH, siendo el más adecuado. Esto se debe a que este solvente a baja concentración, es una disolución acuosa de sales que proceden de ácidos fuertes y bases fuertes, por lo tanto, no se hidroliza y su pH alcanza la neutralidad (Morais, et al, 2010).

El $\mathrm{CaCl}_{2}$ es una sal que proviene de la disociación del ácido clorhídrico y el hidróxido de calcio, su catión calcio divalente $\left(\mathrm{Ca}^{+2}\right)$, no tiene carácter ácido y su anión cloruro $\left(\mathrm{Cl}^{-}\right)$, no posee carácter básico (Ximena et al., 2014). Por lo tanto, no existe la tendencia a aceptar iones hidrógeno $\left(\mathrm{H}^{+}\right)$, tampoco a variar drásticamente los valores de pH. También se observó que cuando se incrementó la concentración del $\mathrm{CaCl}_{2}$ diez veces más, la correlación obtenida fue inversa a la obtenida cuando se empleó como solventes agua y $\mathrm{KCl}$ en las concentraciones de 0.01 y 0.1 molar.

\section{CONCLUSIONES}

Existe dependencia directa entre la medición del $\mathrm{pH}$ y el incremento del volumen de los solventes agua y cloruro de potasio $0.01 \mathrm{M}$ y $0.1 \mathrm{M}$.

No existe dependencia entre la medición del pH y el incremento del volumen, cuando se emplea como solvente al $\mathrm{CaCl}_{2}$ 0.01M. 
Cuando se emplea volúmenes de 100 y 125 y $150 \mathrm{ml}$ del $\mathrm{CaCl}_{2}$ 0.01M y se utiliza compost con tamaños de partícula entre 1 y 2 mm, los valores del pH, tiende a ser homogéneos.

\section{REFERENCIAS BIBLIOGRÁFICAS}

Barrena, R. (2006). Compostaje de residuos sólidos orgánicos... [Universidad Autónoma de Barcelona]. https://www.tdx.cat/bitstream/handle/10803/5307/rbg1de1.pdf

Bohórquez, A., Puentes, J. y Menjivar, J. (2014). Evaluación de la calidad del compost producido a partir de subproductos agroindustriales de caña de azúcar. Corpoica Ciencia y Tecnología Agropecuaria, 15(1), 10. https://www.redalyc.org/pdf/4499/449944863008.pdf

Delgado, J., Valdez, J., y Incio, F. (2019). Influencia de la dilución y concentración de los electrolitos en la medición del pH en suelos ácidos. Revista Científica Pakamuros, 7(2), 13-21. https://doi.org/10.37787/pakamuros-unj.v7i2.89

Florida, N., Reátegui, F., y Pocomucha, V. (2019). Caracterización del Compost a base de plumas de pollos (Gallus gallus domesticus) y otros insumos. RevIA, 6(2), Article 2. https://revistas.unas.edu.pe/index.php/revia/article/view/124

Iqbal, M. K., Shafiq, T., Hussain, A., \& Ahmed, K. (2010). Effect of enrichment on chemical properties of MSW compost. Bioresource Technology, 101(15), 5969-5977. https://doi.org/10.1016/j.biortech.2010.02.105

Lerma H., T. A., Combatt., E. M., \& Palencia L., M. S. (2015). Efecto de la temperatura sobre coloides de suelos agrícolas mediante dispersión dinámica de la luz. Revista de Ciencias Agrícolas, 32(2), 94. https://doi.org/10.22267/rcia.153202.17

Millán, et al (2017) Estudio metodológico sobre la medición de pH y conductividad eléctrica en muestras de compost. Química Orgánica y Bioquímica. Universidad Nacional de Colombia. Revista Colombia Química, página. 247. Bogotá Colombia

Morais, et al (2010) Propiedades ácido-base de las sales. Universidad Politécnica de Valencia. Departamento de Química. Valencia-España

Rivas, M. y Silva R. (2020). Calidad Calidad física y química de tres compost, elaborados con residuos de jardinería, pergamino de café y bora (Eichhornia Crassipes). CIENCIA UNEMI, 13(32), 87100. https://doi.org/10.29076/issn.2528-7737vol13iss32.2020pp87-100p 
Sepúlveda L., y Salinas G. (2014). Evaluación de la calidad química del humus de lombriz roja californiana (Eiseniafoetida) elaborado a partir de cuatro sustratos orgánicos en Arica. Idesia (Arica), 32(2), 95-99. https://doi.org/10.4067/S0718-34292014000200013

Tan, K. (1993) Principles of Soil Chemistry. 2nd ed. Marcel Dekker, Inc., New York, 1993; pp 267 - 271. ISBN: 0-8247-8989-X.

Van Erp, et al. (2001). Relación entre el magnesio extraído por el procedimiento de extracción con cloruro de calcio 0.01 M y los procedimientos convencionales. Comunicaciones en ciencia del suelo y análisis de plantas https://doi.org/10.1081/CSS-100102989

Ximena, D., Bolívar, S., Becerra, H. Q., \& Cataño, D. L. (2014). Evaluación de la formación de ácido clorhídrico a partir de la hidrólisis de las sales inorgánicas en crudos con presencia de ácidos nafténicos Evaluation of hydrochloric acid formation from hydrolysis of inorganic salts in crudes with naphthenic acids co. 28(1), 7-17. 